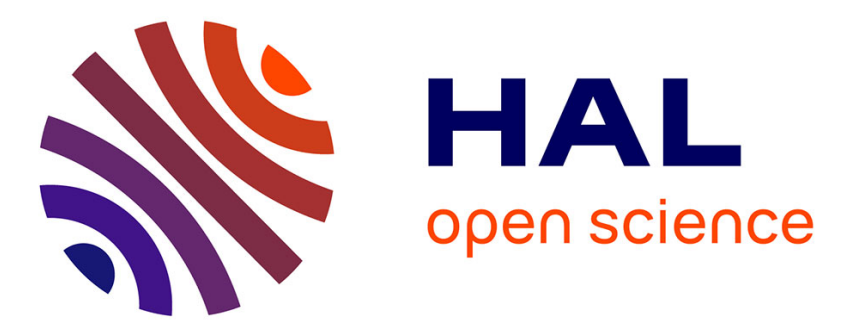

\title{
Power Measurement and Spectral Test of ZigBee Transmitters from 1-bit Under-sampled Acquisition
}

Thibault Vayssade, Florence Azaïs, Laurent Latorre, François Lefevre

\section{To cite this version:}

Thibault Vayssade, Florence Azaïs, Laurent Latorre, François Lefevre. Power Measurement and Spectral Test of ZigBee Transmitters from 1-bit Under-sampled Acquisition. ETS 2019 - 24th IEEE European Test Symposium, May 2019, Baden-Baden, Germany. 10.1109/ETS.2019.8791540 lirmm02274367

\section{HAL Id: lirmm-02274367 https://hal-lirmm.ccsd.cnrs.fr/lirmm-02274367}

Submitted on 29 Aug 2019

HAL is a multi-disciplinary open access archive for the deposit and dissemination of scientific research documents, whether they are published or not. The documents may come from teaching and research institutions in France or abroad, or from public or private research centers.
L'archive ouverte pluridisciplinaire HAL, est destinée au dépôt et à la diffusion de documents scientifiques de niveau recherche, publiés ou non, émanant des établissements d'enseignement et de recherche français ou étrangers, des laboratoires publics ou privés. 


\title{
Power Measurement and Spectral Test of ZigBee Transmitters from 1-bit Under-sampled Acquisition
}

\author{
T. Vayssade ${ }^{(1,2)}$, F. Azaïs ${ }^{(1)}$, L. Latorre ${ }^{(1)}$, F. Lefevre ${ }^{(2)}$ \\ (1) LIRMM, Univ. Montpellier, CNRS, 161 rue Ada, Montpellier, France \\ (2) NXP Semiconductors, 2 Espl. Anton Phillips, 14000 Caen, France
}

\begin{abstract}
This paper presents a low-cost solution to perform power and spectral measurements on RF signals. More specifically, we target RF modulated signals such as $2.4 \mathrm{GHz}$ signals delivered by ZigBee transmitters. The solution is based on 1-bit under-sampled acquisition of the RF signal, acquisition that can be realized using a standard digital ATE channel. The captured binary sequence is then fed to a dedicated postprocessing algorithm, which (i) extracts amplitude and phase variations and (ii) reconstructs the $R F$ signal. From the reconstructed signal, it is possible to perform power and spectral measurements. The different steps of the post-processing algorithm are detailed in the paper. The validity of the technique is demonstrated through both simulations and hardware measurements.
\end{abstract}

Keywords-RF test; ZigBee; digital modulation;OQPSK; 1-bit acquisition, digital signal processing, digital ATE

\section{INTRODUCTION}

Production testing of RF devices is still today a big concern for most of the actors in the semiconductor market [1]. Indeed, the conventional practice relies on the use of an Automatic Test Equipment (ATE) equipped with RF channels, which incurs very high testing costs because such channels are extremely expensive compared to their digital counterparts. Moreover, these resources are usually available in small number, thus reducing multi-site efficiency. An interesting approach to reduce the testing costs is to develop alternative solutions that can be applied on digital ATE. A number of works following this approach can be found in the recent literature targeting RF devices. In [2], a reference transceiver is used to handle test signal generation/reception, accompanied by a FPGA that interfaces the reference transceiver with the digital ATE. In [3], the digital processor embedded in a radio $\mathrm{SoC}$ is used to implement a self-test so that only low-speed digital signals have to be processed by the digital ATE. In [4], a digital ATE system is developed for RF devices with QAM single interfaces based on the concept of direct modulation/demodulation with multilevel drivers and comparators.

In this work, we target a solution for the test of ZigBee transceivers using only a standard digital ATE. A first technique has been proposed in [5] to implement symbol error detection. This solution permits to verify the basic functionality of the device. In this paper, we focus on the problem of performance measurements. More precisely, our objective is to measure the overall power level delivered by the Device Under Test (DUT) and to compute its power spectrum in order verify its compliance with the spectral requirements specified in the IEEE Std 802.15.4 ${ }^{\mathrm{TM}}$.

\section{Signal Under TeSt Characteristics}

This paper targets the development of a low-cost solution for implementing power measurement and spectral test of ZigBee transmitters. All parameters (modulation scheme, spreading method, operating frequency, symbol rate, ...) are set as specified in IEEE Std $802.15 .4^{\mathrm{TM}}$. More specifically, we demonstrate our method in the $2.4 \mathrm{GHz}$ band, which is an ISM band accepted worldwide. Basics of the modulation scheme and the spreading method are briefly given in this section.

In the $2.4 \mathrm{GHz}$ band, the modulation scheme specified by the standard is Offset Quadrature Phase Shift Keying (OQPSK) with half sine pulse shaping. Figure 1 shows the block diagram of a typical implementation. The binary data stream is first split into two branches. Even bits are fed in the in-phase branch (Ibranch) and odd bits in the quadrature-phase branch (Q branch). A bipolar NRZ coding is then applied on each branch, followed by half-sine pulse shaping. A delay of one-bit period (half symbol period) is added on the signal in the $\mathrm{Q}$ branch. The inphase and quadrature-phase components are then modulated onto two orthogonal basis functions, i.e. the I-branch signal is multiplied by a cosine wave and the Q-branch signal by a sine wave. Finally, these two signals are summed to form the OQPSK signal with half-sine pulse shaping.

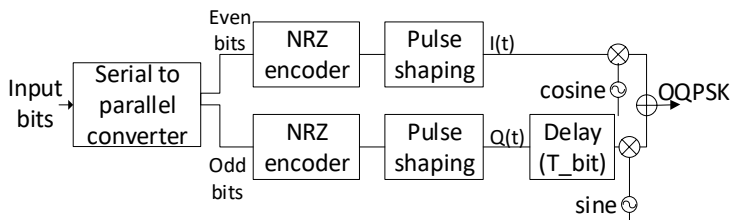

Fig.1: Block diagram of an OQPSK modulator with half-sine pulse shaping

Compared to a classical QPSK scheme, OQPSK with halfsine pulse shaping exhibits enhanced spectral efficiency. Indeed, the additional delay inserted in the Q-branch ensures that signals in the I and Q branches will never change at the same time and limits phase jump to $90^{\circ}$. Moreover, half-sine pulse shaping permits to avoid sharp phase transitions and ensures a constant modulation envelope.

Another feature specified by the standard 802.15 .4 is the use of Direct Sequence Spread Spectrum (DSSS) technique. The general principle consists in multiplying the data being transmitted by a noise signal in order to reduce overall interference. Practically as illustrated in Figure 2, the input binary data are first converted to data symbols, i.e. each octet is divided into two symbols of 4 bits each. With a data rate of 250 $\mathrm{kb} / \mathrm{s}$, the symbol rate is therefore $62.5 \mathrm{ksymb} / \mathrm{s}$. Each data 
symbol is then mapped into one of 16 pseudo-random noise sequences (DSSS sequences), composed of 32 bits (called chips) each. Finally, these chips are fed to the OQPSK modulator. The chip rate is therefore $2 \mathrm{Mchip} / \mathrm{s}$ at the input of the modulator, and $1 \mathrm{Mchip} / \mathrm{s}$ for data in the I- and-Q branches.

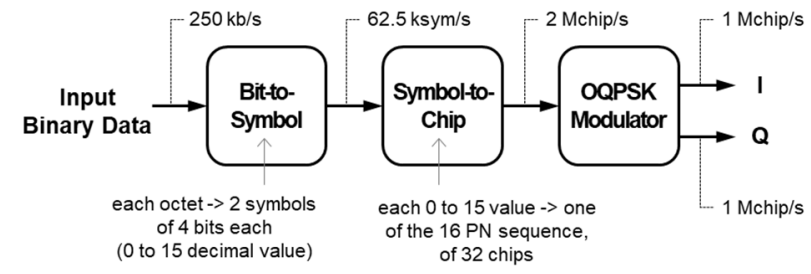

Fig.2: Coding with DSSS technique

\section{TESTING STRATEGY}

The current industrial practice for testing RF devices on the production floor is based on the use of an ATE equipped with RF tester channels. Such channels comprise hardware resources that (i) perform the down-conversion of the RF signal into an analog signal in the Intermediate Frequency (IF) band by mixing the RF signal with a local oscillator and (ii) convert the analog signal in digitized data by using an ADC. Software DSP techniques are then applied on the digitized data stream to compute various features of the signal under test, e.g. code domain sequences, constellation diagram, power spectrum. Due to its high measurement accuracy, the cost of an RF tester channel is extremely high and constitutes a major contributor to the global testing cost of RF devices.

In this context, our strategy to reduce the testing cost of RF devices is to propose new solutions that can be applied using only standard digital ATE channels. The basic principle of the proposed approach is illustrated in Figure 3. The fundamental concept is to use the comparator and the latch comprised in a digital tester channel to perform 1-bit acquisition of the RF signal. During this operation, the amplitude, frequency and/or phase information contained in the analog signal is converted in timing information into the resulting bitstream. The idea is then to develop dedicated post-processing algorithms able to retrieve this information and extract the main signal characteristics.

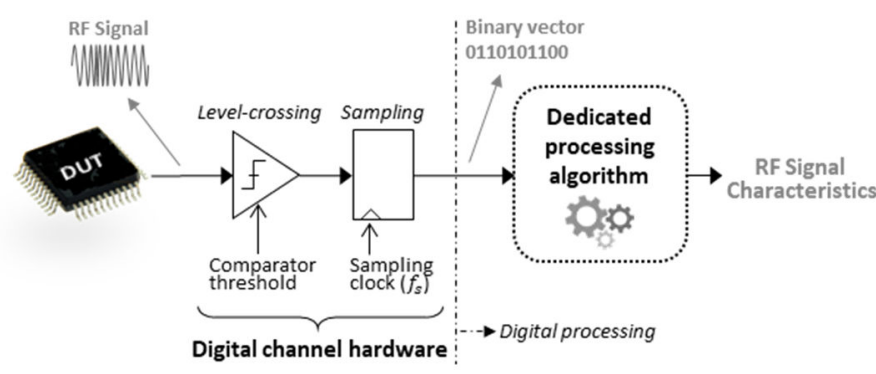

Fig.3: Basic principle of the proposed approach

This strategy of using 1-bit acquisition with a digital tester channel has already been exploited in previous works and dedicated algorithms have been developed for the analysis of AM and FM-modulated signals [6], or to perform phase-noise characterization of signals in the IF frequency band $[7,8]$. However, it is worth noting that these algorithms have been developed based on an oversampled acquisition. In this work, we target $2.4 \mathrm{GHz}$ RF signals, which means that oversampling cannot be used with standard digital tester channel, which typically exhibits a maximum sampling rate at $1.6 \mathrm{Gbps}$.

The solution proposed in this paper relies on 1-bit undersampled acquisition. The principle is derived from the technique exploited in $[9,10]$ to perform precise on-chip timing measurements on high speed digital signals. In those papers, the idea is to sample the high-speed digital signal with a frequency $f_{0}$ by a slightly lower clock frequency $f_{s}$. The resulting signal is a digital signal with a fundamental beat frequency at $f_{b}=$ $f_{0}-f_{s}$. In our practical case, we cannot directly implement this under-sampling scheme because the RF signal frequency at $2.4 \mathrm{GHz}$ exceeds the sampling capabilities of a standard digital tester channel. However, we can use the same principle and sample the signal with a sampling frequency close to a submultiple of the signal frequency $f_{s}=f_{c} / n+f_{d}$, where $f_{d}$ is the frequency deviation from the sub-multiple of the carrier frequency. The resulting signal is a digital signal with a fundamental beat frequency $f_{b}=\left|n f_{s}-f_{c}\right|=n\left|f_{d}\right|$, which contains the information related to amplitude and phase of the original RF signal. Software post-process can then be applied to this low frequency digital signal in order extract the main signal characteristics. Note that in our practical case, the use of $n=2$ is sufficient to satisfy the constraint on the maximum data rate of a standard digital test channel. In the remaining of the paper, we will consider $n=2$.

Based on this approach, a dedicated post-processing algorithm has been developed in [5] that permits to implement symbol error detection. This algorithm involves a phase extraction process associated with a specific demodulation process in order in to retrieve the symbol sequence present in the RF signal. The objective in this paper is to complement this previous work so that we can not only verify the device functionality, but also implement some performance measurements. Specifically, we intend to develop a postprocessing algorithm with a twofold objective: (i) enable the measurement of the power level delivered by the DUT in order to verify its compliance with the datasheet specifications and (ii), enable the measurement of the power spectrum in order to verify its compliance with the spectral requirements specified in the IEEE Std 802.15.4 ${ }^{\mathrm{TM}}$.

Regarding the power level, it is classically expressed in $\mathrm{dBm}$ and computed using the value $(A)$ of either the rms or peak amplitude under the assumption of $50 \Omega$ load impedance:

$$
P_{d B m}=10 \log _{10} \frac{A_{r m s}{ }^{2}}{50 * 1 \mathrm{~mW}}=10 \log _{10} \frac{A_{p k}{ }^{2}}{2 * 50 * 1 \mathrm{~mW}}
$$

The objective of the post-processing algorithm is therefore to be able to extract information on the amplitude level of the RF signal from the binary vector captured by the ATE.

Regarding the power spectrum, it is classically computed by applying an FFT on the time-domain RF signal. The objective of the post-processing algorithm is therefore to be able to reconstruct the time-domain RF signal from the binary vector captured by the ATE. This reconstruction necessitates the evaluation of time-domain fluctuations of the amplitude and phase of the RF modulated signal. 


\section{Post-Processing Algorithm}

Figure 4 gives a general overview of the developed postprocessing algorithm. Details on the different blocks are given in this section.

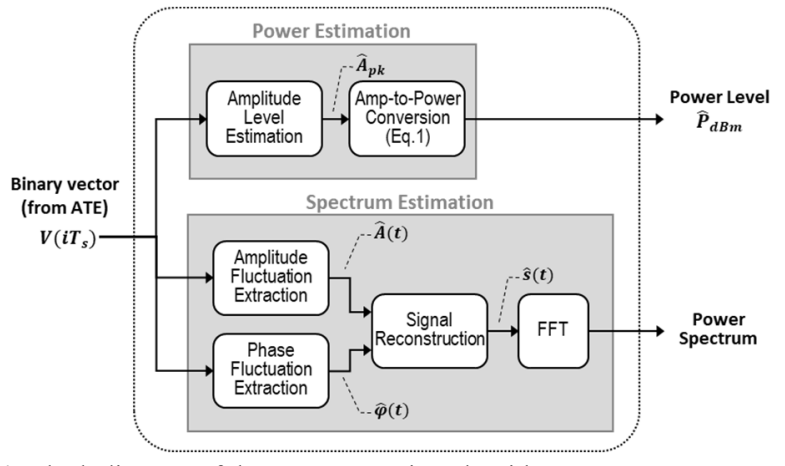

Fig.4: Block diagram of the post-processing algorithm

\section{A. Power level estimation}

The first block of the software post-processing algorithm is the block that realizes the estimation of the RF signal power level. This block first estimates the amplitude level $\hat{A}_{p k}$ and then converts it to power level using Eq.1.

The fundamental basis of the proposed solution comes from the principle of level-crossing that converts voltage-domain information into timing information. To illustrate this point, let us consider a sine-wave that is converted into a square-wave by level crossing (with a comparator threshold $C$ different than 0 ), as illustrated in Figure 5. It can be easily shown that there is a direct relationship between the sine-wave peak amplitude $A_{p k}$ and the square-wave duty cycle $\alpha=\Delta T / T$ [6]:

$$
A_{p k}=\frac{C}{\cos \left(\pi \frac{\Delta T}{T}\right)}
$$

where $\Delta T$ is duration between the rising and falling transitions of the square-wave signal and $T$ is the period of both the sinewave and square-wave signals.

This equation indicates that it is possible to evaluate the amplitude of a sine-wave signal simply by measuring the duty cycle of the square-wave signal generated with level-crossing.

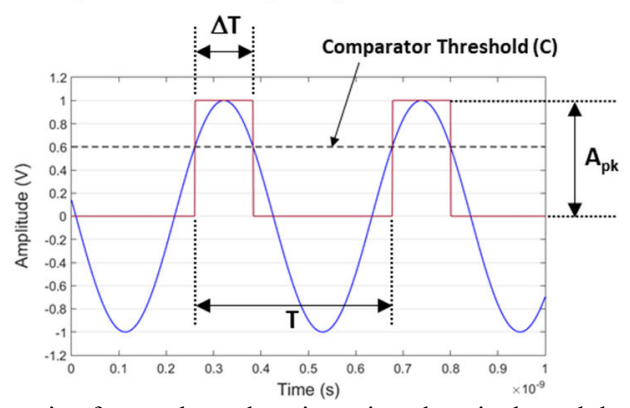

Fig.5: Conversion from voltage domain to time domain through level-crossing

In our context, the binary vector captured by the ATE can be considered as a square-wave signal that results from a level crossing operation combined with an under-sampling process. The under-sampling process translates the signal towards a lower frequency but preserves its essential characteristics. Therefore, the relationship between the RF signal amplitude and the digital signal duty cycle is still valid. In other words, by measuring the average duty cycle of the digital signal, we can have an estimation of the average signal amplitude.

This is the solution that has been implemented in the postprocessing algorithm. Practically, the measure of the average duty cycle is directly derived from the binary vector captured by the ATE by counting the ratio between the number samples at logic "1" over the total number of samples. An estimation of the mean value of the peak amplitude is then given by:

$$
\hat{A}_{p k}=\frac{C}{\cos \left(\pi * \frac{\text { Nbof } " 1 "}{\text { Nb of samples }}\right)}
$$

Note that the accuracy achieved in the amplitude estimation depends on the level of the comparator threshold. As established in [6], the higher the comparator threshold with respect to the signal amplitude, the better the accuracy. However, the comparator threshold should not be set too close to the signal amplitude for noise robustness reasons. Practically in this paper, the comparator threshold is set at $70 \%$ of the nominal signal amplitude, which is a good compromise between accuracy and noise robustness.

\section{B. Spectrum estimation}

The second block of the software post-processing algorithm is the block that realizes the estimation of the power spectrum. Different tasks are involved in this process as detailed hereafter.

\section{1) Amplitude fluctuation extraction}

A first task is to retrieve the amplitude variation in the RF signal envelope. The fundamental basis of the proposed solution is the same than for amplitude level estimation, but in this case we are interested in the time-domain fluctuations of the RF signal amplitude. Note that although an OQPSKmodulated signal with half-sine pulse shaping normally exhibits a constant modulation envelope, the presence of imperfections in the transmitter chain may create some amplitude fluctuations. In particular, one of the most important contributor to these amplitude fluctuations is the presence of IQ imbalance, which creates a variation in the RF signal envelope at the chip rate of $1 \mathrm{MHz}$. It is therefore essential to be able to evaluate such lowfrequency variations.

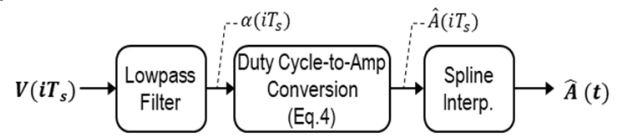

Fig.6: Amplitude fluctuation extraction from the binary vector captured by the ATE

In this context, the idea is to monitor the variations over the time of the digital signal duty cycle in order to evaluate amplitude fluctuations in the envelope of the RF signal. Figure 6 depicts the practical implementation. The measurement of the duty cycle $\alpha=\Delta T / T$ is realized by applying a low-pass filter on the captured digital signal $V\left(i T_{s}\right)$. The filter should be designed such that it passes the components related to the low-frequency variations of the RF signal envelope, i.e. with a bandwidth greater than the chip rate of $1 \mathrm{MHz}$, but eliminates all components related to the square waveform of the digital signal, i.e. with a cut-off frequency lower than the fundamental beat frequency. In this work, we have chosen a Chebyshev filter of type II with stopband starting at $7 \mathrm{MHz}$. The output of the filter is a discrete-time signal 
$\alpha\left(i T_{s}\right)$ whose value is representative of the digital signal duty cycle. Duty cycle to amplitude conversion is then simply realized based on Eq.2:

$$
\hat{A}\left(i T_{S}\right)=\frac{C}{\cos \left(\pi \alpha\left(i T_{S}\right)\right)}
$$

This signal gives an estimation of the time-domain fluctuations of the RF signal envelope. Spline interpolation is finally applied to obtain a signal $\hat{A}(t)$ with a finer time resolution for the subsequent step of signal reconstruction.

\section{2) Phase fluctuation extraction}

In parallel of amplitude fluctuation extraction, a second task is to retrieve the phase variation of the RF modulated signal. The proposed solution is based on the work proposed in [5] and involves several operations, as depicted in Figure 7.

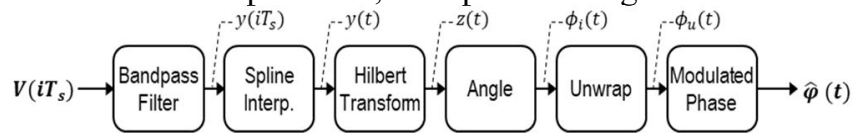

Fig.7: Phase fluctuation extraction from the binary vector captured by the ATE

The captured digital signal $V\left(i T_{s}\right)$ is first filtered by with a band-pass filter centered on the square wave beat frequency $f_{b}=\left|2 f_{s}-f_{c}\right|$ in order to eliminate the harmonics introduced by 1-bit quantization. The resulting signal is a discrete-time sine-wave signal $y\left(i T_{s}\right)$. Spline interpolation is applied to this signal in order to increase its time resolution, so that it can be considered as a continuous-time signal $y(t)$. The instantaneous phase $\Phi_{i}(t)$ is then derived from the Hilbert Transform of the signal with:

$$
\Phi_{i}(t)=\operatorname{atan}\left(\frac{H T[y(t)]}{y(t)}\right)
$$

Due to the atan function, the computed instantaneous phase is a wrapped phase. By unwrapping $\Phi_{i}(t)$, we obtain a linear evolution of the instantaneous phase $\Phi_{u}(t)$, which can be expressed as:

$$
\Phi_{u}(t)=2 \pi f_{b} t+\varphi(t)
$$

where the first term corresponds to the linear phase of a signal with a fundamental beat frequency $f_{b}$ and the second term actually corresponds to the phase fluctuation $\varphi(t)$ of the modulated RF signal.

Finally using the expression of the fundamental beat frequency, an estimation of the phase fluctuation of the RF modulated signal is given by:

$$
\hat{\varphi}(t)=\Phi_{u}(t)-2 \pi\left|2 f_{s}-f_{c}\right| t
$$

\section{3) Signal reconstruction and FFT}

The last task is to reconstruct the RF modulated signal and compute its spectrum. Signal reconstruction is performed by adding the estimated amplitude fluctuation $\hat{A}(t)$ and the estimated phase fluctuation $\hat{\varphi}(t)$ to an ideal RF sine-wave at carrier frequency:

$$
\hat{s}(t)=\hat{A}(t) \cdot \sin \left(2 \pi f_{c} t \pm \hat{\varphi}(t)\right)
$$

Note that in this expression, the delay introduced by the filters used in amplitude and phase extraction are compensated. Indeed, the filter used for amplitude extraction introduces a delay different than the filter used for phase extraction. Also note that depending whether the sampling frequency is chosen with a positive or negative deviation with respect to the carrier half-frequency, the estimated phase should be added or subtracted to take into account spectral inversion. In particular, $\hat{\varphi}(t)$ must be subtracted if $f_{s}>f_{c} / n$, and added otherwise.

Finally, once the RF signal is reconstructed, its power spectrum is simply derived from classical FFT computation.

\section{VALIDATION}

The software post-processing has been implemented in Matlab/Simulink ${ }^{\circledR}$. It has been validated first in simulation and then demonstrated through hardware measurements. Results are presented in this section.

\section{A. Simulation results}

To validate the technique, we have considered a simulated signal generated using the model of Figure 1 for the Device Under Test. The generated RF signal is an OQPSK modulated signal with a carrier frequency $f_{c}=2.48 \mathrm{GHz}$ (channel 26 in ZigBee) and a nominal amplitude of $1 \mathrm{~V}$. The digital ATE channel has been modeled by a level-crossing operation (threshold set at $70 \%$ the nominal signal amplitude) followed by a sampling operation with a frequency $f_{s}=1.25 \mathrm{GHz}$. The resulting signal is therefore a square-wave signal with a fundamental beat frequency $f_{b}=20 \mathrm{MHz}$. This signal is used as the input of the post-processing algorithm.

First, we have considered an ideal signal without any imperfections. The result of the phase extraction process is shown in Figure 8, which compares the phase of the RF signal generated by the OQPSK modulator to the phase reconstructed from the 1-bit under-sampled acquisition. A very good agreement is observed between both curves, with a difference that remains below $2^{\circ}$ and an rms error of only $0.95^{\circ}$.
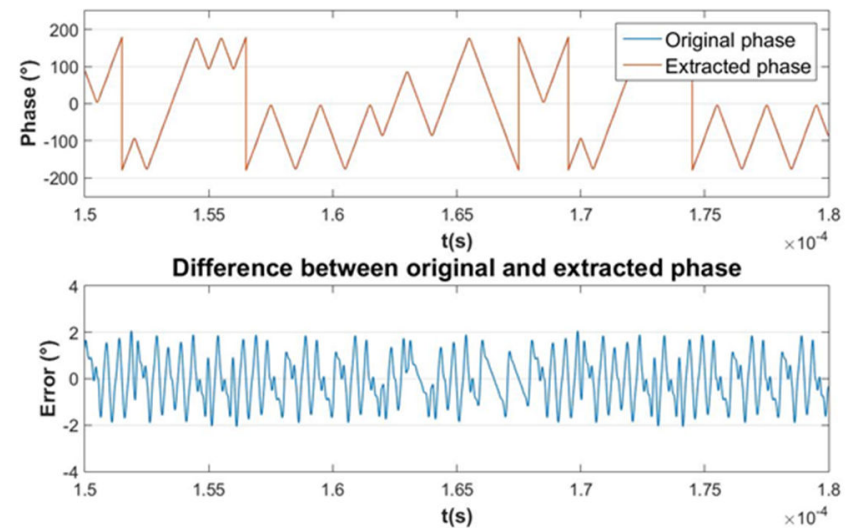

Fig.8: Comparison between original and reconstructed phase for a simulated perfect RF signal

Then, we have considered a simulated signal that presents low-frequency variations in the RF signal amplitude. For this, we have injected $10 \%$ IQ imbalance in the model of the OQSPK modulator. The result of the envelope extraction process is shown in Figure 9, which compares the original envelope of the RF signal and the reconstructed one obtained from 1-bit acquisition. A very good agreement can be observed between both curves. In particular, it can be observed that the reconstructed envelope is well-centered on the original one and that the difference between both curves does not exceed $15 \mathrm{mV}$. 


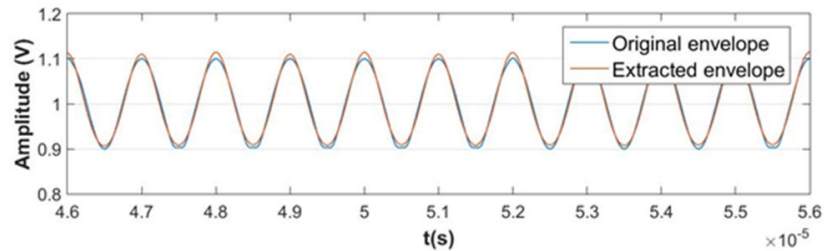

$\mathbf{t}(\mathrm{s})$

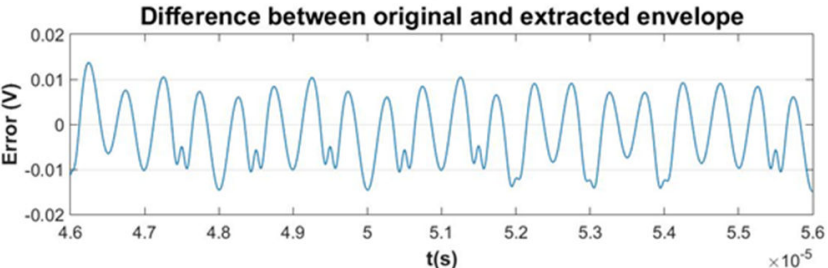

$\mathrm{t}(\mathrm{s})$

Fig.9: Comparison between original and reconstructed envelope for a simulated RF signal with $10 \%$ IQ imbalance

To further validate the envelope extraction process, we have performed additional simulations varying the level of injected IQ imbalance between $0 \%$ up to $30 \%$. Results are summarized in Figure 10, which reports the rms error between the original envelope and the reconstructed one versus the level of injected IQ imbalance. It can be observed that the rms error increases as the level of IQ imbalance increases. However, even in presence of an IQ imbalance as large as $30 \%$, the rms error remains below $90 \mathrm{mV}$. These results therefore indicate that correct reconstruction of the RF signal envelope can be achieved in a large range of amplitude fluctuations.

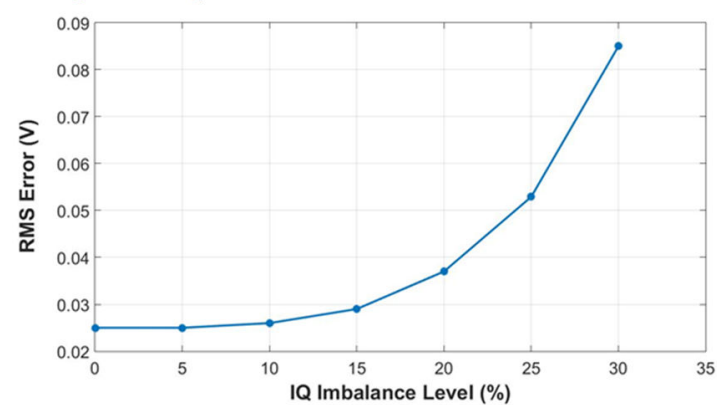

Fig.10: Rms error between original and reconstructed envelope vs. the level of injected IQ imbalance.

Finally, to validate the ability of the technique to enable spectral measurements, we have compared the power spectrum of the reconstructed RF signal to the power spectrum of the simulated original RF signal. As illustrated in Figure 11, results show a very good agreement between both spectrums.

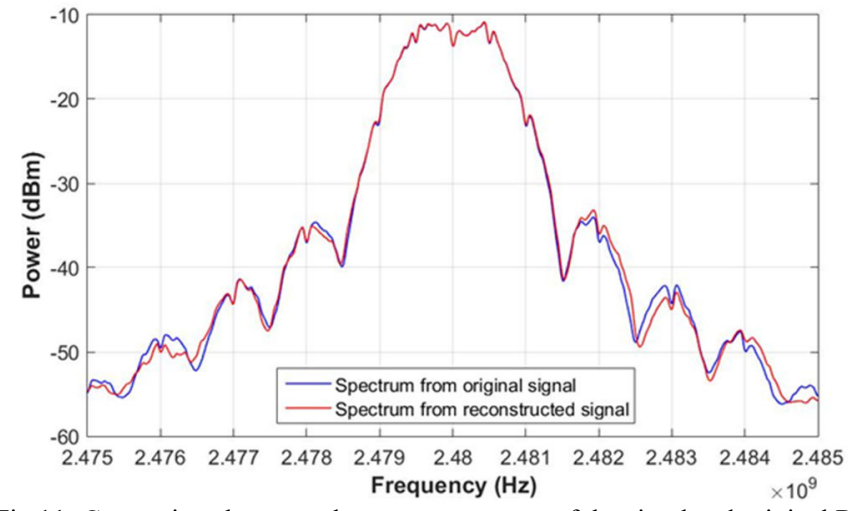

Fig.11: Comparison between the power spectrum of the simulated original RF signal and the power spectrum of the reconstructed RF signal

\section{B. Hardware measurement results}

To corroborate simulation results by hardware measurements, the experimental setup depicted in Figure 12 has been developed. A Universal Software Radio Peripheral (USRP) is used to emulate the Device Under Test. The interest of using an USRP is that it enables the possibility to include imperfections such as IQ imbalance or phase noise in the generation of the signal. The RF signal generated by the USRP is then divided in two signals by using a resistive power divider. The first output of the power divider is directly connected to a Digital Storage Oscilloscope (DSO). The second output of the power divider is passed through a latched comparator (ADCMP 572 of Analog Devices) that emulates the digital tester channel by converting the RF modulated signal into a square-wave signal. This "binary" signal is connected to a second channel of the oscilloscope. Both signals acquired by the oscilloscope are then transferred to a PC; the post-processing algorithm is applied on the binary signal and results are compared with the regular measurements performed on the RF signal.

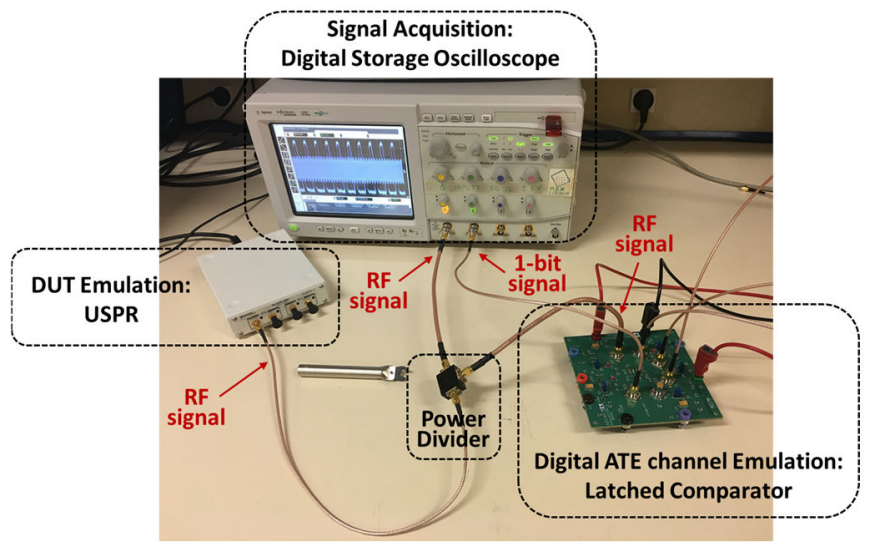

Fig.12: Experimental setup for hardware measurements

In this demonstration, we use the same conditions as for simulation, i.e. the USRP is programmed to generate an RF signal with a carrier frequency $f_{c}=2.48 \mathrm{GHz}$ and the sampling frequency of the latched comparator is set at $f_{s}=1.25 \mathrm{GHz}$. The output of the latched comparator is then a binary signal with a fundamental beat frequency $f_{b}=20 \mathrm{MHz}$. The acquisition at the oscilloscope is performed at 5Gsamples/s, which satisfies the Nyquist rate for the RF signal. The complete acquisition is conserved for the analysis of the RF signal while decimation by 4 is applied on the binary signal in order to keep only 1.25 Gsamples/s. Note that the setup does not provide the best conditions because there is no synchronization between the sampling clock of the latched comparator and the oscilloscope.

Results of the phase and amplitude extraction processes are illustrated in Figure 13 and 14, respectively. In both cases, there is a good match between the curve measured on the full RF signal and the one reconstructed from the under-sampled 1-bit signal. The rms error is $1.39^{\circ}$ for the phase extraction, and $24 \mathrm{mV}$ for the amplitude extraction, which is in correct agreement with simulation results. Note that for the amplitude extraction, a 10\% IQ imbalance has been inserted in the USRP in order to have low-frequency variations in the envelope of the generated RF signal. 


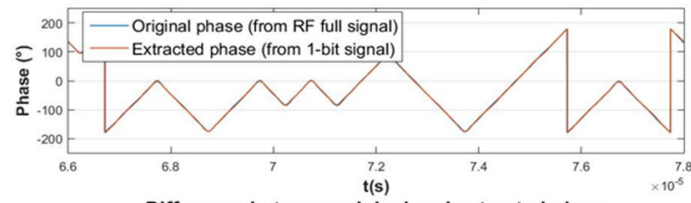

Difference between original and extracted phase

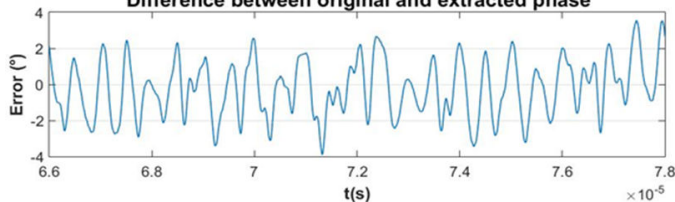

inal and reconstructed phase for an Fig.13: Comparison between original an
experimental RF signal without IQ imbalance
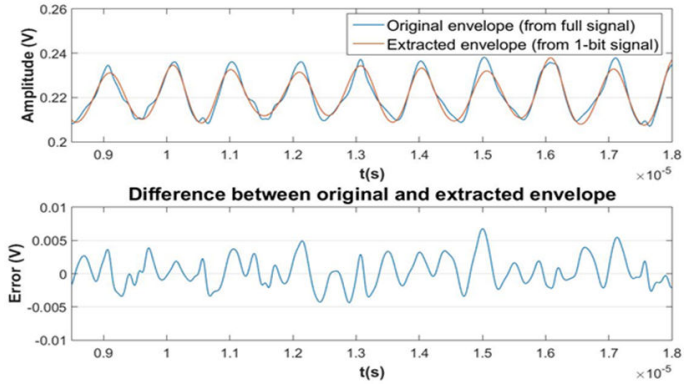

Fig.14: Comparison between original and reconstructed envelope for an experimental RF signal with 10\% IQ imbalance

These results validate the ability of the technique to perform accurate estimations of the amplitude and phase fluctuation. From these estimations, we can reconstruct the RF signal and compute its spectrum. Figure 15 compares the spectrum computed directly on the RF signal acquired by the oscilloscope and the one computed on the reconstructed RF signal after processing of the 1-bit signal. Again, very good agreement is observed between both spectrums, demonstrating that the quality of the reconstructed RF signal is appropriate for a correct evaluation of the power spectrum.

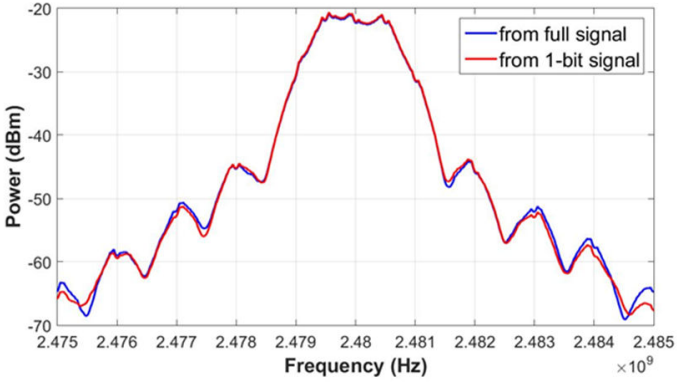

Fig.15: Comparison between the power spectrum computed on the original RF signal and the power spectrum computed on the reconstructed RF signal

Finally, we have verified the ability of the technique to perform power measurements on various levels of power. Practically, we have varied the power level (i.e. the signal amplitude) of the RF modulated signal generated by the USRP. For each level, power has been measured on the full RF signal on one hand, and estimated from the 1-bit signal on the other hand. Results are reported in Figure 16, which plots the power level estimated on the 1-bit signal versus the power level measured on the RF signal. It can be clearly observed that the proposed solution offers excellent match over a broad range of power levels, with a maximum error between both measurements that remains below $0.3 \mathrm{dBm}$.

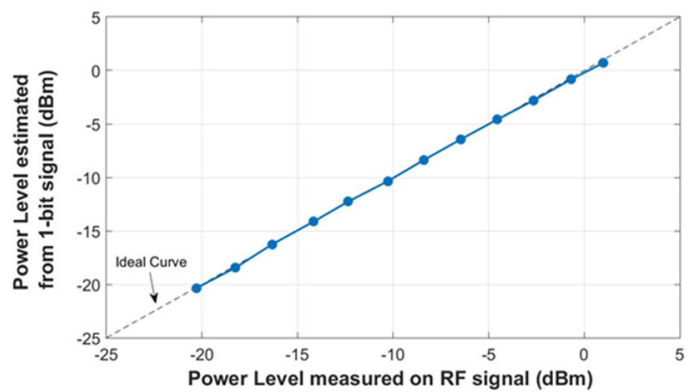

Fig.16: Power level estimated from 1-bit signal vs. power level measured on the RF signal

\section{CONCLUSION}

In this paper, we have developed a low-cost solution for implementing power measurement and spectral test of ZigBee transmitters using only a standard digital ATE. The technique relies 1-bit under-sampled acquisition of the RF signal with a sampling frequency close to half the carrier frequency. A dedicated post-process algorithm is then applied on captured binary data which permits to (i) estimate the power level and, (ii) reconstruct the RF modulated signal and obtain its power spectrum. The technique has been validated on $2.4 \mathrm{GHz} \mathrm{ZigBee}$ simulated signals and on actual physical signals but can be applied to any band limited RF signal. The developed postprocessing algorithm also constitutes a first step towards EVM measurement, which is another essential performance to be verified during production test. Future work will concentrate on this aspect so that we can propose the implementation of a complete test flow on a standard digital ATE.

\section{ACKNOWLEDGMENT}

This work has been carried out under the framework of PENTA-EUREKA project HADES.

\section{REFERENCES}

[1] F. Demmerle, "Integrated RF-CMOS Transceivers challenge RF Test," Proc. IEEE Int'1 Test Conf., p. 8, 2006.

[2] I. Kore et al., "Multi-site test of RF transceivers on low-cost digital ATE," Proc. IEEE International Test Conference (ITC), pp. 1-10, 2011.

[3] C. H. Peng et al., "A novel RF self test for a combo SoC on digital ATE with multi-site applications," Proc. Int'l Test Conf. (ITC), p. 8, 2014.

[4] M. Ishida K. Ichiyama, "An ATE System for Testing RF Digital Communication Devices With QAM Signal Interfaces," IEEE Design \& Test, vol. 33, no. 6, pp. 15-22, 2016.

[5] T. Vayssade et al., "Low-cost functional test of a $2.4 \mathrm{GHz}$ OQPSK transmitter using standard digital ATE”, Proc. IEEE Int'l On-Line Test Conferance (IOLTS), p.6, 2018.

[6] N. Pous et al., J.,"A Level-Crossing Approach for the Analysis of RF Modulated Signals using only Digital Test Resources", J. of Electronic Testing: Theory and App. (JETTA), vol. 27, no. 3, pp 289-303, 2011

[7] S. David-Grignot et al., "Low-cost phase noise testing of complex RF ICs using standard digital ATE," Proc. IEEE Int'l Test Conf. (ITC), p. 9, 2014.

[8] S. David-Grignot et al., "A new technique for low-cost phase noise production testing from 1-bit signal acquisition," Proc.IEEE European Test Symposium (ETS), p.6, 2015.

[9] J-L Huang, K-T Cheng, "An on-chip short-time interval measurement technique for testing high-speed communication links," Proc. IEEE VLSI Test Symposium (VTS), pp. 380-385, 2001.

[10] S. Sunter, A. Roy, "On-chip digital jitter measurement, from megahertz to gigahertz," IEEE Design \& Test of Computers, vol. 21, no. 4, pp. 314-321, 2004. 